\title{
Hypoglycaemia Remains the Key Obstacle to Optimal Glycaemic Control - Continuous Glucose Monitoring is the Solution
}

\author{
Peter Adolfsson, ${ }^{1,2}$ Donald Rentoul, ${ }^{3}$ Brigitte Klinkenbij| ${ }^{4}$ and Christopher G Parkin ${ }^{4}$ \\ 1. Department of Pediatrics, Kungsbacka Hospital, Sweden; 2. Institute of Clinical Sciences, The Sahlgrenska Academy, University of Gothenburg, Sweden; \\ 3. Dexcom, Edinburgh, Scotland; 4. CGParkin Communications, Inc., Henderson, NV, US
}

W e queried PubMed and other internet databases to identify studies, meta-analyses, review articles and other data sources regarding hypoglycaemia incidence/costs/impacts and continuous glucose monitoring (CGM) use. Our analysis of the evidence showed that hypoglycaemia remains a significant health concern and a primary obstacle to optimal adherence to prescribed diabetes treatment. In addition to its adverse clinical consequences, hypoglycaemia negatively impacts quality of life and places additional financial burdens on patients, patient families, employers and healthcare payers. Clinical trials have shown that the use of CGM can reduce the incidence and duration of hypoglycaemic episodes. This article reviews relevant CGM studies, discusses the prevalence and clinical/ financial implications of hypoglycaemia, and explores the strengths and limitations of current CGM systems in minimising the burden of hypoglycaemia.

\section{Keywords}

Hypoglycaemia, nocturnal hypoglycaemia, impaired hypoglycaemia awareness, continuous glucose monitoring, quality of life

Disclosures: Peter Adolfsson has received a research grant from Novo Nordisk and consulting fees associated with lectures/meetings from Eli Lilly, Novo Nordisk, Abbott, Dexcom, Medtronic, Nordic Infucare, Roche and Rubin Medical. Christopher Parkin has received consulting fees from CeQur, Dexcom, Insulet, Johnson \& Johnson, Mannkind, and Roche Diabetes Care and Senseonics. Donald Rentoul and Brigitte Klinkenbijl are employees of Dexcom. Review Process: Double-blind review.

Authorship: All named authors meet the International Committee of Medical Journal Editors (ICMJE) criteria for authorship of this manuscript, take responsibility for the integrity of the work as a whole, and have given final approval to the version to be published.

open Access: This article is published under the Creative Commons Attribution Noncommercial License, which permits any non-commercial use, distribution, adaptation and reproduction provided the original author(s) and source are given appropriate credit. (c) The Authors 2018.

\section{Received: 19 June 2018}

Accepted: 17 July 2018

Citation: European Endocrinology, 2018;14(2):50-6 Corresponding Author: Christopher G Parkin, CGParkin Communications, Inc., 2352 Martinique, Henderson, NV 89044, USA. E: chris@cgparkin.org

Support: Funding for the development of this manuscript was provided by Dexcom, Inc., San Diego, CA, USA.
Intensive insulin therapy is recommended for the management of type 1 diabetes (T1D), ${ }^{1}$ and has been shown to be an effective and beneficial treatment modality for many individuals with type 2 diabetes (T2D).,3 During the past decade, we have seen several new insulin preparations and delivery devices that enable individuals with diabetes to achieve near-normal glycaemia. However, despite advances in insulin therapy and ways to deliver insulin, a large proportion of individuals with insulin-treated diabetes are still not achieving their treatment goals. ${ }^{4,5}$

Suboptimal treatment adherence is often identified as the underlying cause of poor diabetes control. However, adherence is often driven by several factors over which patients have little control, such as cost, complexity of dosing regimens, patient-provider interaction and others. ${ }^{6}$ Moreover, anecdotal evidence suggests that many patients with diabetes do not know how to identify and interpret their glucose monitoring results or how their medications and health behaviours actually influence their glucose levels. ${ }^{7.8}$

Although these factors clearly play a major role in suboptimal treatment adherence, hypoglycaemia remains a key obstacle to achieving desired glycaemic control in insulin-treated diabetes. ${ }^{9}$ Numerous studies have shown that many individuals with insulin-treated diabetes are unable to meet their glycaemic targets without excessive and/or severe hypoglycaemia. ${ }^{9-11}$

Recent advances in continuous glucose monitoring (CGM) technologies may now empower individuals with insulin-treated diabetes to minimise or avoid severe hypoglycaemia as they achieve desired glycaemic control. In this article, we review the adverse clinical and financial consequences of severe hypoglycaemia and discuss current options for the use of CGM, real-time CGM (rtCGM) and intermittent scanning CGM (iSCGM) - by individuals with insulin-treated diabetes to safely manage their diabetes.

\section{What do we know?}

Several recent studies have demonstrated the clinical utility of CGM in reducing hypoglycaemia in T1D ${ }^{12-17}$ and multiple daily insulins injection-treated T2D. 18,19 Because most of these studies have been reviewed in the literature, further comment on them is not needed. However, findings from the HypoDE, ${ }^{16} \mathrm{GOLD}-3^{17}$ and IMPACT (subgroup analysis) ${ }^{20}$ studies are relevant to our discussion and provide important insights into the value of CGM specific to hypoglycaemia reduction and prevention.

In the HypoDE study, which included patients with MDI-treated T1D with problematic hypoglycaemia (severe hypoglycaemia and/or impaired hypoglycaemia awareness), investigators found that the number of hypoglycaemic events can be markedly reduced by the use of rtCGM compared with 
reliance on conventional blood glucose monitoring. ${ }^{16}$ Investigators also reported that rtCGM use resulted in a significant decrease in the frequency of clinical severe hypoglycaemia and reduced glycaemic variability, a known risk factor for hypoglycaemia. .1,22 $^{2}$

The GOLD-3 study looked at the effects of rtCGM within a cohort of patients with MDI-treated T1D with varying degrees of hypoglycaemia. ${ }^{17}$ Study outcomes included time in nocturnal hypoglycaemia $(<70 \mathrm{mg} / \mathrm{dL}$ $[<3.9 \mathrm{mmol} / \mathrm{L}]$ and $<54 \mathrm{mg} / \mathrm{dL}[<3.0 \mathrm{mmol} / \mathrm{L}])$, number of nocturnal and daytime hypoglycaemic events, and daytime hypoglycaemia and hypoglycaemia confidence, as measured by the Hypoglycaemia Confidence Scale. ${ }^{23}$ Investigators observed significant reductions in nocturnal and daytime hypoglycaemia among rtCGM users compared with blood glucose monitoring. For participants randomised to start with rtCGM, the time in hypoglycaemia decreased during rtCGM use but increased when rtCGM use was stopped prior to the washout period. In addition, rtCGM users reported increased confidence in their ability to detect and respond to impending hypoglycaemia. This finding has positive clinical implications, given the relationship between the fear of hypoglycaemia and adherence to suboptimal medication..$^{24,25}$

The IMPACT study assessed the impact of Freestyle Libre isCGM system (Abbott, Chicago, IL, US) use on hypoglycaemia compared with capillary glucose monitoring in individuals with well-controlled (glycated haemoglobin [HbA1c] $\leq 7.5 \%$ [58 mmol/mol] $)$ T1D treated with MDI therapy - individuals with impaired hypoglycaemia awareness were excluded. ${ }^{13}$ Participants in the intervention group used Freestyle Libre throughout the study and could obtain flash glucose readings during the final 14-day period and adjust their blood glucose control accordingly, whereas the control group used self-monitoring of blood glucose (SMBG) and wore a blinded Freestyle Libre during the final 14-day period. A pre-specified subgroup analysis showed that the use of the Freestyle Libre system significantly reduced time in hypoglycaemia without deterioration of $\mathrm{HbA} 1 \mathrm{C}$, and improved treatment satisfaction, with the proviso that the individuals scanned their device frequently (average of 15 times a day). ${ }^{20}$ The significance of these findings were questioned by Warren, ${ }^{26}$ noting that 'it would be extremely surprising if individuals who could see their flash glucose levels did not keep them in target more effectively than blinded individuals'. It is our view that the results reported in the IMPACT study simply show that Freestyle Libre is a better option than SMBG but requires frequent scanning to reduce hypoglycaemia. Recently, the authors of the IMPACT trial responded that the methodology was indeed noted in the original article, but that there was no other practical alternative to this approach. ${ }^{27}$

However, the degree of hypoglycaemia reduction achieved varies between CGM systems. The recent IHART trial, a head-to-head comparative study, demonstrated that the use of the Dexcom G5 system more effectively reduces the time spent in hypoglycaemia than with Freestyle Libre use in MDI-treated T1D adults with impaired hypoglycaemia awareness. ${ }^{28}$

\section{Why do we care?}

\section{Hypoglycaemia is prevalent}

The latest estimates of the frequency of hypoglycaemia range from 42-91 events per patient-year for T1D adults and from 20.3-44.4 events per patient-year for T2D adults. ${ }^{29}$ Data from the T1D Exchange registry indicate that approximately $11.8 \%$ of adults and $6.2 \%$ of children/ adolescents experience at least one episode of severe hypoglycaemia per year. ${ }^{30,31}$ In a 2005 survey by Akram et al., 16.5\% of 401 patients reported at least one severe hypoglycaemic event within the past year. ${ }^{32}$ In contrast, lower frequency of severe hypoglycaemia has been reported in Sweden where 60\% of children used an insulin pump, $80 \%$ used a CGM device and only $2.1 \%$ had a severe hypoglycaemia event during 2016. ${ }^{33}$

Key contributors to severe hypoglycaemia are asymptomatic hypoglycaemia (impaired hypoglycaemia awareness) and nocturnal hypoglycaemia. ${ }^{34}$ Both conditions impede patients' perception of hypoglycaemia when it is occurring and, thus, limits their ability to take appropriate action. Impaired hypoglycaemia awareness results from frequent hypoglycaemic events (severe and non-severe). ${ }^{35}$ Nocturnal hypoglycaemia accounts for a significant proportion of hypoglycaemic events and often goes undetected. ${ }^{36}$ In an early study of 70 patients using CGM, data revealed unrecognised hypoglycaemia in $62.5 \%$ patients with T1D and $46.6 \%$ of patients with T2D $-73.7 \%$ of all episodes occurred during the night. ${ }^{37}$ Nocturnal hypoglycaemia is particularly concerning among adults and children after physical exercise. ${ }^{38,39}$ As reported by Gomez et al., the risk of hypoglycaemia is elevated for at least 24 hours in recovery from exercise, with the greatest risk of nocturnal hypoglycaemia occurring after afternoon activity. ${ }^{38}$

Among adults with T1D, severe hypoglycaemia is more related to long duration of diabetes and socioeconomic status than $\mathrm{HbA} 1 \mathrm{C}^{30}$ whereas factors such as being non-white, no health insurance and lower household income were associated with higher frequency of severe hypoglycaemia among T1D children/adolescents. ${ }^{31}$ Conversely, severe hypoglycaemia is most common in T2D adults (treated with insulin or secretagogues) with impaired hypoglycaemia awareness. ${ }^{32}$

Severe hypoglycaemia is particularly concerning among elderly patients with diabetes. These patients are at significantly higher risk due to their age, diabetes duration, insulin therapy duration, glucose variability and higher prevalence of impaired hypoglycaemia awarewness. ${ }^{10,11,40-42}$ cognitive and physical impairments and other co-morbidities further increase the risk of severe hypoglycaemia among older patients. ${ }^{42}$

Although targeting lower $\mathrm{HbA} 1 \mathrm{c}$ has been cited as a key contributor to severe hypoglycaemia, an analysis of the T1D Exchange data showed that this condition is common in adults with T1D with both higher and lower HbA1c levels. ${ }^{30}$ Moreover, national data from Sweden show that it is possible to improve both $\mathrm{HbA1C}$ and reduce severe hypoglycaemia with insulin pump and CGM use - however, the technology has to be used properly. ${ }^{33}$ Severe hypoglycaemia is also common among older adults with T2D across all levels of glycaemic control. The risk tends to be higher in patients with either near-normal glycaemia or very poor glycaemic control. ${ }^{43}$

\section{New cut-points for hypoglycaemia}

Cut-points and definitions of hypoglycaemia have been debated over the years. However, an international panel of clinicians and researchers with expertise in CGM technologies recently published consensus recommendations for classifying and defining hypoglycaemia from data derived from CGM use. ${ }^{44}$ The panel described three levels of hypoglycaemia (Table 1) and recommended that hypoglycaemia should be quantified in the following ways: percentage of time spent in the hypoglycaemic ranges ( $<70-54 \mathrm{mg} / \mathrm{dL}[<3.9-3.0 \mathrm{mmol} / \mathrm{L}]$ or $<54 \mathrm{mg} / \mathrm{dL}[<3.0 \mathrm{mmol} / \mathrm{L}])$, the number of minutes or hours below these levels, and the number of hypoglycaemic events that occur over the given CGM reporting period. ${ }^{44}$ An event was defined as any episode of hypoglycaemia that lasts $\geq 15$ minutes. Glucose levels $\geq 70 \mathrm{mg} / \mathrm{dL}$ ( $3.9 \mathrm{mmol} / \mathrm{L}) \geq 15$ minutes indicate that the event has ended. 
Table 1: Classifications of hypoglycaemia ${ }^{44}$

\begin{tabular}{|l|l|}
\hline $\begin{array}{l}\text { Level 1: Non-severe } \\
<70-54 \mathrm{mg} / \mathrm{dL}(3.9-3.0 \mathrm{mmol} / \mathrm{L}) \text { with or without symptoms }\end{array}$ & $\begin{array}{l}\text { - Should be considered an alert that the individual may be at risk for developing hypoglycaemia and } \\
\text { should work to minimise the time spent in this range to reduce the risk of developing more clinically } \\
\text { significant hypoglycaemia. }\end{array}$ \\
\hline $\begin{array}{l}\text { Level 2: Significant } \\
<54 \mathrm{mg} / \mathrm{dL}(3.0 \mathrm{mmol} / \mathrm{L}) \text { with/without symptoms }\end{array}$ & - Should be considered clinically significant hypoglycaemia, requiring immediate attention. \\
\hline $\begin{array}{l}\text { Level 3: Severe } \\
\text { Not defined by a specific glucose value }\end{array}$ & - Denotes cognitive impairment requiring external assistance for recovery. \\
\hline
\end{tabular}

\section{Hypoglycaemia impacts lives Clinical consequences}

The clinical consequences of severe hypoglycaemia are well known and include: precipitation of acute cerebrovascular disease; myocardial infarction; neurocognitive dysfunction; and loss of vision. ${ }^{44}$ Left untreated, severe hypoglycaemia can result in significant morbidity and mortality. ${ }^{66,47}$

As discussed earlier, nocturnal hypoglycaemia is a significant risk factor for severe hypoglycaemia and is thought to be linked to sudden death during sleep. ${ }^{48}$ Although asymptomatic, nocturnal hypoglycaemia can trigger sleep disturbances in patients, resulting in morning headache, chronic fatigue and/or mood changes. ${ }^{45}$

Severe hypoglycaemia is extremely problematic in paediatric patients, often resulting in convulsions or enuresis during nocturnal hypoglycaemic episodes. ${ }^{49}$ It is also linked to long-term cognitive impairment in paediatric patients with T1D. A 16-year follow-up study found that T1D children who experienced severe hypoglycaemia at an early age ( $\leq 10$ years) had poorer cognitive function in adulthood, and that deficits were found across several cognitive domains, particularly among patients exposed to severe hypoglycaemia before the age of 6 years. ${ }^{50}$ Some studies have reported decrements in the domains of intelligence quotient (IQ), executive functions, delayed memory and processing speed in childhood-onset T1D. However, these decrements are often not observed until the children are studied later in childhood. ${ }^{51}$ It has been suggested that 'chronic exposure to different aspects of dysglycemia is additive, and that brain and cognitive changes only become apparent over time'. ${ }^{51}$

\section{Hypoglycaemia fear}

The frequency of severe hypoglycaemic episodes is a central driver of hypoglycaemia fear, ${ }^{9,52-54}$ which can result in suboptimal insulin treatment adherence and adverse health outcomes. ${ }^{24,25}$ Patients who are fearful of hypoglycaemia are often reluctant to adhere to their prescribed insulin regimens, 9,55 leaving them at high risk for developing the microvascular and macrovascular complications of hyperglycaemia. ${ }^{1,56-58} \mathrm{~A}$ recent international survey of 27,585 patients with diabetes found that reducing insulin doses in response to hypoglycaemia was common, ranging from $33.6-51.7 \%$ in patients with T1D and from $25.8-46.7 \%$ in patients with T2D. ${ }^{29}$ Moreover, parental fears can prompt 'hypoglycaemia avoidance' behaviours, which lead to poor glycaemic control and increased risk of long-term complications. ${ }^{25,59}$

\section{Economic impact}

The direct costs of treating severe hypoglycaemia (emergency department care and/or hospitalisations) vary from country to country. Relatively recent data from Germany, Spain and the UK estimate the average costs per event to be $€ 441$, $€ 577$ and $€ 236$, respectively, for patients with T1D, and $€ 533$, $€ 691$ and $€ 537$, respectively, for patients with T2D. ${ }^{60}$ Costs are much higher in the USA, with an average cost of $\$ 1,387$ per event. ${ }^{61}$
However, all levels of hypoglycaemia (non-severe, significant, severe) also confer significant indirect costs on employers as well as individuals with diabetes. ${ }^{62,63} \mathrm{~A}$ recent study showed a clear link between severe hypoglycaemia and the costs of lost productivity. ${ }^{62}$ According to the report, mean annualised indirect costs due to lost productivity rose with increasing severity of hypoglycaemia: $\$ 7,248, \$ 7,493$ and $\$ 12,167$, respectively.62 The adverse economic impact on patients due to increased out-of-pocket expenses, decreased productivity and lost workdays is equally notable, with the highest loss in productivity attributable to nonsevere nocturnal hypoglycaemic events. ${ }^{63}$

\section{Human impact}

Although the economic consequences of hypoglycaemia are, indeed, significant, they are overshadowed by its adverse effects on patients' lives. Numerous studies have shown that hypoglycaemia negatively impacts patients' ability to concentrate and participate in daily activities, $^{64}$ thereby negatively impacting patients' quality of life. ${ }^{65}$ Patients who experience frequent episodes of hypoglycaemia often feel powerless, creating anxiety for themselves and their families, and mood swings, irritability and feelings of depression are common. ${ }^{66,67}$ A key contributor to diminished quality of life is chronic sleep interruption caused by nocturnal hypoglycaemia, itself, and fear of hypoglycaemia. 68.69 A recent survey by Barnard et al. found that chronic sleep disruption is common among patients and parents/caregivers, and is associated with detrimental impacts on virtually all aspects of daily life. ${ }^{.8}$

Even non-severe hypoglycaemia, which occurs in $24-60 \%$ of patients with diabetes, can adversely affect quality of life. The greatest reductions in quality of life are seen among those participants reporting a higher frequency of non-severe hypoglycaemia. ${ }^{65}$ As reported by GeelhoedDuijvestijn et al., it takes an average of 50.4 minutes to return to normal functioning following a daytime non-severe hypoglycaemic event, but negative feelings persisted for an average of 5.4 hours. $^{70}$ Following a nocturnal non-severe hypoglycaemic event, functionality was diminished for an average of 80.5 minutes and negative feelings persisted for 12.2 hours. ${ }^{70}$

Hypoglycaemia at all levels places an additional layer of stress and frustration on individuals with diabetes who are already burdened with the complexities of daily diabetes management. It is, therefore, gratifying that recent studies have shown that isCGM, as well as rtCGM, use improves glycaemic outcomes and quality of life in patients with $T 1 D^{12-17,20}$ and patients with T2D treated with intensive insulin therapy ${ }^{18,19}$ regardless of hypoglycaemia incidence and severity. However, it is important that patients and their clinicians understand the strengths and limitations of various CGM systems in order to select the most appropriate system that meets their individual needs.

\section{Which CGM features are most important?}

Patients who choose to use CGM have several options available to them, standalone devices or insulin pump systems with integrated CGM. 
Table 2: Current continuous glucose monitoring systems

\begin{tabular}{|l|l|l|}
\hline System & Manufacturer & Minimum age for use \\
\hline FreeStyle Libre & Abbott Diabetes Care, Alameda, USA & $\geq 18$ (US), $\geq 4$ (ex-US) \\
\hline Guardian 3 & Medtronic, Inc., Northridge, USA & $\geq 14$ \\
\hline Dexcom G5 & Dexcom, Inc., San Diego, USA & $\geq 2$ \\
\hline Dexcom G6 & Dexcom, Inc., San Diego, USA & $\geq 2$ \\
\hline Eversense & Senseonics, Inc., Germantown, USA & $\geq 18$ \\
\hline
\end{tabular}

Table 3: Comparison of key system attributes relevant to hypoglycaemia

\begin{tabular}{|c|c|c|c|c|c|}
\hline & isCGM & rtCGM & & & \\
\hline Feature & Freestyle Libre & Dexcom G6 & Dexcom G5 ${ }^{\dagger}$ & Guardian 3 & Eversense \\
\hline $\begin{array}{l}\text { Accuracy } \\
\text { Overall MARD, \% } \\
\text { Low-glucose MARD, \% } \\
\quad \text { Range: } \mathrm{mg} / \mathrm{dL}(\mathrm{mmol} / \mathrm{L}) \\
\text { Low-glucose MAD, } \mathrm{mg} / \mathrm{dL}(\mathrm{mmol} / \mathrm{L}) \\
\quad \text { Range: } \mathrm{mg} / \mathrm{dL} \text { (mmol/L) } \\
\text { Percentage of matched CGM-reference pairs in adults (low glucose) } \\
\quad \text { Ranges, } \mathrm{mg} / \mathrm{dL} \text { (mmol/L) } \\
\quad \geq 40-60(\geq 2.2-3.3) \\
\quad>60-80(>3.3-4.4)\end{array}$ & $\begin{array}{l}9.772 \\
24.077 \\
\leq 70(\leq 3.9) \\
13.0(0.7)^{77} \\
\leq 70(\leq 3.9)\end{array}$ & $\begin{array}{l}9.073 \\
-\S \\
11.5(0.6)^{73} \\
54-<70(3.0-<3.9)\end{array}$ & $\begin{array}{l}9.074 \\
-\S \\
6.7(0.5)^{74} \\
61-80(3.4-4.4) \\
\\
74 \%^{78} \\
68 \%^{78}\end{array}$ & $\begin{array}{l}10.475 \\
9.475 \\
61-80(3.4-4.4) \\
\\
-\end{array}$ & $\begin{array}{l}8.876 \\
9.076 \\
55-70(3.1-3.9) \\
-\end{array}$ \\
\hline Automatic data transmission & no & Yes & yes & yes & yes \\
\hline Active alarms & no & Yes & yes & yes & yes \\
\hline Real-time data sharing & no & Yes & yes & yes & yes \\
\hline
\end{tabular}

*Data unavailable.

¿Dexcom lower-glucose range data is only reported as MAD.

${ }^{+}$Data are from accuracy assessment of the Dexcom G4 sensor with 505 Software 505, which is now used in the Dexcom G5 system.

FData are derived from US Food and Drug Administration documents (Summary of Safety and Effectiveness Data [SSED]); data for the Dexcom G6 and Eversense sensors are not. yet available.

MAD = Mean Absolute Difference (between sensor readings and reference values, expressed as $\mathrm{mg} / \mathrm{dL}$ ); MARD = Mean Absolute Relative Difference (between sensor readings and reference values, expressed as \%); isCGM = intermittently scanned continuous glucose monitoring; rtCGM = real-time continuous glucose monitoring

Because some integrated pump systems (Medtronic 640G and 670G) address hypoglycaemia by automatically suspending or reducing insulin infusion, we have focused only on current systems, which can be used as standalone devices or other insulin pump systems (Table 2). There are distinct differences between systems in terms of accuracy, indications for insulin dosing, alarms and alerts, wear time, convenience features and cost.

We recently published suggestions for the appropriate selection of CGM devices based upon matching the various CGM systems to individual patients' needs for daily self-management. ${ }^{11}$ In this article, we narrow our focus to the specific system attributes that are most relevant to addressing hypoglycaemia: accuracy in the hypoglycaemic ranges, continuous automatic transmission of glucose data, active audible alarms, and data sharing (Table 3).

\section{Accuracy in hypoglycaemic range}

Accuracy in all glycaemic ranges is required for the safe and effective use of CGM. Although the overall accuracy (mean absolute relative difference) of the various devices is mostly similar (Table 3), accurate and reliable data in hypoglycaemic ranges is particularly critical. The obvious danger is that failure to detect hypoglycaemia will likely result in an acute (potentially severe) hypoglycaemic event. Conversely, an erroneous low-glucose reading may prompt users to correct with food, resulting in avoidable hyperglycaemia. Importantly, persistence in CGM use is impacted by patients' perceptions of the accuracy and reliability of their CGM devices. ${ }^{79}$
Inaccuracy in the lower-glucose ranges is a characteristic of all current CGM devices. As shown in Table 3, the overall accuracy of the Dexcom, Libre and Eversense systems is relatively similar, however lower overall accuracy has been observed with the Guardian 3. Differences in accuracy are also seen in the lower-glycaemic ranges. The accuracy of the Libre and Dexcom G6 in the lower ranges are comparable when measured by mean absolute difference, however comparison of matched CGMreference pairs in the lowest range $(\geq 40-60 \mathrm{mg} / \mathrm{dL}[\geq 2.2-3.3 \mathrm{mmol} / \mathrm{L}])$ show much lower CGM-reference value concurrence with the Freestyle Libre system. Abbott Diabetes Care addresses this issue by advising Freestyle Libre users to perform a confirmatory finger-stick test when sensor values are in the low-glucose range.

Differences in study designs, methodologies, metrics and other factors make it difficult to draw direct comparisons between systems, however the substantial difference between the Freestyle Libre and rtCGM systems (as demonstrated using standardised methodology) cannot be ignored. Concerns about inaccuracies of the Freestyle Libre system were recently reported by Fokkert et al., who observed a lowglucose bias in the lower ranges and underestimation of postprandial glucose response. ${ }^{77}$

Although lower accuracy in the lower-glycaemic ranges is common in all systems, reduction or prevention of hypoglycaemia can be achieved by taking action before the level of hypoglycaemia is reached. Automatic audible alerts in the rtCGM devices provide additive protection when glucose values decrease rapidly. 
Table 4: Open-ended responses regarding real-time data sharing80

'Not only has it given me more peace of mind ... it has given her peace of mind to know that someone else is watching and worrying about her blood sugar so she doesn't have to. She can be a carefree kid again (kind of).'

'It is amazing how much it has changed our lives. Our child has so much more freedom now! ' Continuous glucose monitoring is a powerful tool in managing my child's diabetes ... continuous glucose monitoring allows her to have more independence with her peers.'

'I think it's helpful for babysitters and home caregivers. At school, I leave it to the school nurse because she takes excellent care of my child and we touch base daily.'

'I like the layer of awareness it gives to others. If his alarm for a low goes off on my phone while he is at school, I can see if he is being addressed.'

\section{Automatic data transmission}

All current CGM systems continuously monitor glucose levels in interstitial fluid. However, an important difference between the Freestyle Libre system and the other systems is how data are delivered to the user. With the Dexcom G6, Dexcom G5, Guardian 3 and Eversense systems, glucose data are transmitted automatically to the user's receiver and/or smartphone in real time.

\section{Active alarms}

Active audible alerts and alarms automatically warn rtCGM users when glucose levels rise or fall beyond a defined glycaemic threshold or when glucose values rapidly increase or decreases beyond a defined threshold (e.g., predictive alerts). Beyond this, trend arrows indicate both the direction and velocity of changing glucose, thus enabling users to take appropriate pre-emptive action to prevent impending glycaemic events. This around-the-clock vigilance is particularly important for patients with frequent severe hypoglycaemia, nocturnal hypoglycaemia and/or impaired hypoglycaemia awareness.

The Dexcom G5, Dexcom G6, Guardian 3 and Eversense systems feature programmable active audible alarms. The Dexcom G5 and G6 Mobile App feature allows users to schedule and customise a second group of alarms that only apply during the hours specified (e.g., work hours). The Guardian 3 system features customised predictive alarms - from 10 minutes up to 1 hour - allowing users additional time to respond as needed. The Eversense system provides on-body vibration alarms when an alert level has been reached and triggers the mobile device app to sound an alert and display messages on the screen.

The Freestyle Libre does not offer automatic alarms. Instead, users must scan frequently to receive information about actual glucose value, an 8-hour glucose history and trend arrows. The individual must then rely on their ability to recognise impending and acute hypoglycaemia.

\section{Real-time data sharing}

The ability to share real-time glucose data with family members, friends and caregivers provides a potent safety net for all CGM users who are vulnerable to severe hypoglycaemia, such as adults who travel frequently and elderly patients who are at increased risk for severe hypoglycaemia due to impaired awareness ${ }^{10,11,40-42}$ and/or cognitive and physical impairments. ${ }^{42}$

The use of real-time data sharing is particularly valuable in paediatric patients because it allows parents to monitor their child's glycaemic status at school, during physical activity and throughout the night. This not only provides an added layer of safety for the child, but also supports better overall diabetes management by reducing hypoglycaemia fear among parents. Moreover, rtCGM offers the ability to achieve good nocturnal glucose control while receiving an alert/alarm in case the glucose value passes a predefined level. Therefore, parents/guardians can sleep well during most nights while being warned during the nights when needed. Thus, rtCGM even improves health in a more general way.

In a recent study, Erie et al. surveyed parents and daytime caregivers of 33 paediatric patients with T1D who used a CGM device (Dexcom or Medtronic) with real-time data sharing. ${ }^{80}$ Fifty-seven survey pairs were distributed, and 33 parent surveys and 17 daytime caregiver surveys were returned to the investigators. Results showed that all parents and $78 \%$ of caregivers felt that use of the data-sharing feature decreased their worry or stress. Table 4 presents some of the parent/caregiver open-ended responses reported in the study.

All current CGM systems offer data-sharing abilities but with distinct differences in functionality. The Dexcom G5/G6, Eversense and Guardian 3 systems allow sharing of real-time glucose levels and trends to up to five 'followers', whereas the Freestyle Libre system allows sharing with up to 20 followers.

There are differences in the type of data that is transferred. The Dexcom G5 and G6 systems provide current glucose values, trend arrows and trend graphs. The Eversense system shares the current glucose value and trend arrow. With the Guardian 3 system, the follower receives a message and must then connect to the web browser + follower function (via the CareLink Connect service) to investigate the reason behind the alert. The Freestyle LibreLinkUp app provides current glucose values and trend arrows, however it requires users to scan the sensor in order to send glucose data to followers. This significantly limits the follower's ability to detect and warn the patient of impending or acute severe hypoglycaemia when the patient is sleeping. The same kind of restriction applies to physical exercise which is a situation associated to both hyper- and hypoglycaemia. Although scanning is a quick and simple procedure, this product attribute does not allow the use of active alarms and continuous, automatic real-time data-sharing, which we feel are critically important features relative to hypoglycaemia detection/prevention and patient safety.

\section{Recommendations}

All of the current CGM systems provide significant advantages over traditional blood-glucose monitoring. A key strength of the Freestyle Libre or isCGM is that the system is easy to start with, intuitive to function and marketed at a relatively low price. Thus, it is a much more effective and easier way to measure glucose levels compared to SMBG. For patients without problematic hypoglycaemia, a number of factors (e.g., convenience, cost, ease-of-use) should of course be considered when selecting a CGM system. However, for patients who are at increased risk of severe hypoglycaemia, the attributes discussed here - accuracy in the low-glucose range, automatic data transmission, active alerts/ alarms and real-time data sharing - should be priority considerations. 
As shown in Table 2, accuracy in the lower-glucose ranges is an issue for all current systems, and some systems perform better than others. It is our view that active alarms 'trump' accuracy in prevention and detection for patients with frequent non-severe hypoglycaemia, frequent severe hypoglycaemia, nocturnal hypoglycaemia and/or impaired awareness. An early warning of impending hypoglycaemia provides the opportunity to take corrective action to prevent a severe event. The ability to share data with others in real time is another important safety feature that should be considered.

The Dexcom G5, Dexcom G6, Guardian 3 and Eversense systems offer these protections. Although frequent scanning with the Freestyle Libre system provides some protection during the day, it cannot detect nocturnal hypoglycaemia when the user (or caregiver) is sleeping or warn the physically active individual about a pending hypoglycaemia. Therefore, it is our recommendation that the use of the Freestyle Libre in patients with problematic hypoglycaemia should only be considered when cost or other factors prohibit the use of a real-time CGM system. However, when considering the cost of a specific device, it is important that both patients and healthcare payers factor in the known costs of hypoglycaemia as they relate to clinical risk and consequences, increased hospitalisations and emergency care, diminished productivity, additional financial burden on patients and poor quality of life. When viewed from this perspective, initial cost of a device is far outweighed by total cost if the device does not provide adequate protection against hypoglycaemia. In some European countries, the prescription of rtCGM is required for individuals with impaired awareness or other problematic hypoglycaemia, and they are not eligible for isCGM reimbursement.

\section{Summary}

Severe hypoglycaemia poses a significant health risk and is a costly complication for patients with MDI-treated diabetes. It is particularly dangerous in patients with impaired hypoglycaemic awareness and when it occurs during the night. The fear of severe hypoglycaemia and resulting hypoglycaemia are key drivers of suboptimal adherence to prescribed insulin therapy, which can lead to increased risk for long-term microvascular and macrovascular disease.
Although inaccuracy in the lower glucose ranges remains a limitation for all current CGM systems, devices that provide automatic 'predictive' alerts before glucose falls into the hypoglycaemic range mitigates this limitation. Automatic sharing of this predictive information with family members, friends and/or caregivers further reduces the risk of both moderate and severe hypoglycaemic events.

In addition to improvements in HbA1C and patients' quality of life, current CGM technologies enable patients to detect and prevent severe hypoglycaemia. However, given the different functionalities and varying levels of protection provided by current CGM systems, it is important that patients and their clinicians carefully consider the strengths, weaknesses and appropriateness of each system as they apply to individual needs.

Numerous recent studies have demonstrated the clinical efficacy and other benefits of CGM use in individuals with T1D and T2D regardless of the insulin delivery method used. ${ }^{12,14,18,81-85}$ Driven by advances in accuracy, functionality and usability, CGM adoption has increased dramatically over the past decade, and its use should be encouraged in all insulin-treated patients. As advances in CGM technology continue, we anticipate even greater accuracy and more convenience features in future CGM systems.

Moreover, we will see increasing adoption of data visualisation/ interpretation tools such as the Ambulatory Glucose Profile (AGP), which automatically calculates a number of key metrics - time in glucose ranges (low, target, high), estimated HbA1c (eHbA1c) and glycemic variability. With AGP information, in combination with the individual day-profiles (which can include medication, carbohydrateintake, exercise and other event details), healthcare professionals can more effectively collaborate with their patients as they review the data, discuss problematic issues and agree upon solutions that are individualised to the needs of each patient. Thus, CGM technologies may contribute greatly to minimising the important 'hypoglycaemia' side effect of intensive insulin therapy, and improve both outcomes and quality of life for people with diabetes. $\square$
1. The Diabetes Control and Complications Trial Research Group. The effect of intensive treatment of diabetes on the development and progression of long-term complications in insulin-dependent and progression of long-term complications in ins

2. Li Y, Xu W, Liao Z, et al. Induction of long-term glycemic control in newly diagnosed type 2 diabetic patients is associated with improvement of $\beta$-cell function. Diabetes Care. 2004;27:2597-602

3. Weng J, Li Y, Xu W, et al. Effect of intensive insulin therapy on $\beta$-cell function and glycaemic control in patients with newly diagnosed type 2 diabetes: a multicentre randomised parallelgroup trial. The Lancet. 2008;371:1753-60.

4. Carls G, Huynh J, Tuttle E, et al. Achievement of glycated hemoglobin goals in the US remains unchanged through 2014. Diabetes Ther. 2017;8:863-73.

5. Miller KM, Foster NC, Beck RW, et al. T1D Exchange Clinic Network. Current state of type 1 diabetes treatment in the U.S. updated data from the T1D Exchange clinic registry. Diabetes Care. 2015:38:971-8

6. Garcia-Perez LE, Alvarez M, Dilla T, Gil-Guillen V, Orozco-Beltran D. Adherence to therapies in patients with type 2 diabetes. Diabetes Ther Res TreatEduc Diabetes Rel Dis. 2013;4:175-94.

Silva DD, Bosco AA. An educational program for insulin selfadjustment associated with structured self-monitoring of blood glucose significantly improves glycemic control in patients
with type 2 diabetes mellitus after 12 weeks: a randomized, controlled pilot study. Diabetol Metab Syndr. 2015;7:2.

8. Cypress M, Tomky D. Using self-monitoring of blood glucose in noninsulin-treated type 2 diabetes. Diabetes Spectrum. 2013;26:102-6.

9. Cryer PE. Hypoglycemia: still the limiting factor in the glycemic management of diabetes. EndocrPract. 2008;14:750-6.

10. Giorda $\mathrm{CB}$, Ozzello $\mathrm{A}$, Gentile $\mathrm{S}$, et al. Incidence and risk factors for severe and symptomatic hypoglycemia in type 1 diabetes: for severe and symptomatic hypoglycemia in type 1 diabetes:
results of the HYPOS-1 study. Acta Diabetol. 2015:52:845-53.

11. Cariou B, Fontaine P, Eschwege $E$, et al. Frequency and Cariou B, Fontaine $\mathrm{P}$, Eschwege $\mathrm{E}$, et al. Frequency and
predictors of confirmed hypoglycaemia in type 1 and insulin-treated type 2 diabetes mellitus patients in a real-life setting: results from the DIALOG study. Diabetes Metab. 2015;41:116-25.
12. Beck RW, Riddlesworth T, Ruedy K et al. Effect of continuous glucose monitoring on glycemic control in adults with type 1 diabetes using insulin injections: the DIAMOND randomized diabetes using insulin injections: the
clinical trial. JAMA. 2017:317:371-8.

13. Bolinder J, Antuna R, Geelhoed-Duijvestijn P, et al. Novel glucose-sensing technology and hypoglycemia in type 1 diabetes: a multicentre, non-masked, randomised controlled trial. Lancet. 2016;388:2254-63.

14. Riddlesworth T, Price D, Cohen N, Beck RW. Hypoglycemic event frequency and the effect of continuous glucose monitoring in adults with type 1 diabetes using multiple daily insulin injections. Diabetes Ther. 2017;8:947-51.

15. Ruedy KJ, Parkin CG, Riddlesworth TD, Graham C, for the DIAMOND Study Group.Continuous glucose monitoring in older adults with type 1 and type 2 diabetes using multiple daily injections of insulin: results from the DIAMOND trial. J Diabetes injections of insulin: results from
Sci Technol. 2017;11:1138-46.

16. Heinemann L, Freckmann G, Ehrmann D, et al. Real-time continuous glucose monitoring in adults with type 1 diabetes and impaired hypoglycaemia awareness or sever diabetes and impaired hypoglycaemia awareness or severe
hypoglycaemia treated with multiple daily insulin injections (HypoDE): a multicentre, randomised controlled trial. Lancet. 2018;391:1367-77.

17. Ólafsdóttir AF, Polonsky WH, Bolinder J, et al. A randomized clinical trial of the effect of continuous glucose monitoring on nocturnal hypoglycemia, daytime hypoglycemia, glycemic variability, and hypoglycemia confidence in persons with type 1 diabetes treated with multiple daily insulin injections (GOLD-3) Diabetes Technol Ther. 2018;20:274-84.

18. Beck RW, Riddlesworth TD, Ruedy K, et al. Continuous glucose monitoring versus usual care in patients with type 2 diabetes receiving multiple daily insulin injections: a randomized trial. Ann Intern Med. 2017;167:365-74

19. Haak T, Hanaire H, Aijan R, et al. Flash glucose-sensing technology as a replacement for blood glucose monitoring for the management of insulin-treated type 2 diabetes: a multicenter, open-label randomized controlled trial. Diabetes Ther. 2017;8:55-73.

20. Oskarsson P, Antuna R, Geelhoed-Duijvestijn P, et al. Impact of flash glucose monitoring on hypoglycaemia in adults with type 1 diabetes managed with multiple daily injection therapy: a pre-specified subgroup analysis of the IMPACT randomised a pre-specified subgroup analysis of the IMPACT

21. Monnier L, Wojtusciszyn A, Colette C, Owens D. The contribution of glucose variability to asymptomatic hypoglycemia in persons with type 2 diabetes. Diabetes Technol Ther. 2011;13:813-8.

22. Qu Y, Jacober $S$, Zhang $Q$, et al. Rate of hypoglycemia in insulin- treated patients with type 2 diabetes can be predicted from glycemic variability data. Diabetes Technol Ther. 2012;14:1008-219.

23. Polonsky WH, Fisher L, Hessler D, Edelman SV. Investigating hypoglycemic confidence in type 1 and type 2 diabetes. Diabetes Technol Ther. 2017;19:131-6.

24. Wild $D$, von Maltzahn $R$, Brohan $E$, et al. A critical review of the literature on fear of hypoglycemia in diabetes: implications for literature on fear of hypoglycemia in diabetes: Implications for
diabetes management and patient education. Patient Educ diabetes management
couns. 2007;68:10-5.

25. Haugstvedt A, Wentzel-Larsen T, Graue M, et al. Fear of hypoglycaemia in mothers and fathers of children with type 1 diabetes is associated with poor glycaemic control and parental emotional distress: a population-based study. Diabet Med. 2010;27:72-8.

26. Warren RE. Flash glucose monitoring: objective, not selfreferential, outcomes are needed. Diabetologia. https://doi. org/10.1007/s00125-018-4608-0 (accessed 2 May 2018).

27. Bolinder J, Oskarsson P, Antuna R, et al. Flash glucose monitoring: objective, not self-referential, outcomes are needed. Reply to Warren RE [letter]. Diabetologia. https://doi. org/10.1007/s00125-018-4652-9. Accessed May 29, 2018.

28. Reddy M, Jugnee N, El Laboudi A, et al. A randomised controlled pilot study of continuous glucose monitoring and flash glucose pilot study of continuous glucose monitoring and flash gl
monitoring in people with type 1 diabetes and impaired awareness of hypoglycaemia. Diabet Med. 2018;35:483-90.

29. Khunti K, Alsifri S, Aronson R, et al. Impact of hypoglycaemia on patient-reported outcomes from a global, 24-country study of 27,585 people with type 1 and insulin-treated type 2 diabetes. Diabetes Res Clin Pract. 2017;130:121-9. 
30. Weinstock RS, Xing D, Maahs DM, et al.; T1D Exchange ClinicNetwork. Severe hypoglycemia and diabetic ketoacidosis in adults with type 1 diabetes: results from the T1D Exchange clinic registry. J Clin Endocrinol Metab. 2013;98:3411-9.

31. Cengiz E, Xing D, Wong JC, et al. Severe hypoglycemia and diabetic ketoacidosis among youth with type 1 diabetes in the T1D Exchange Clinic Registry. Pediatr Diabetes. 2013; 14: 447-54.

32. Akram K, Pedersen-Bjergaard U, Carstensen B, et al. Frequency and risk factors of severe hypoglycaemia in insulin-treated Type 2 diabetes: a cross-sectional survey. Diabet Med. 2006;23:750-6.

33. Swediabkids Annual Report 2016. https://www.diabethics.com/ forskning-teknik/swediabkids-2016/. Accessed May 23, 2018.

34. Unger J, Parkin CG. Hypoglycemia in insulin-treated diabetes: a case for increased vigilance. Postgrad Med. 2011;123:81-91.

35. Kenny C. When hypoglycemia is not obvious: diagnosing and treating under-recognized and undisclosed hypoglycemia. Prim Care Diabetes. 2014:8:3-11.

36. Jauch-Chara K, Schultes B. Sleep and the response to hypoglycemia. Best Pract Res Clin Endocrinol Metab. 2010;24:801-15.

37. Chico A, Vidal-Rios P, Subira M, Novials A. The continuous glucose monitoring system is useful for detecting unrecognized hypoglycemias in patients with type 1 and type 2 diabetes but is not better than frequent capillary glucose measurements for improving metabolic control. Diabetes Care. 2003;26:1153-7.

38. Gomez AM, Gomez C, Aschner P, et al. Effects of performing morning versus afternoon exercise on glycemic control and hypoglycemia frequency in type 1 diabetes patients on sensoraugmented insulin pump therapy. J Diabetes Sci Technol. 2015;9:619-24.

39. The Diabetes Research in Children Network (DirecNet) Study Group. Impact of exercise on overnight glycemic control in children with type 1 diabetes J Pediatr. 2005:147:528-34

40. Weinstock RS, DuBose SN, Bergenstal RM, et al. Risk factors associated with severe hypoglycemia in older adults with type 1 diabetes. Diabetes Care. 2016;39:603-10.

41. Bremer JP, Jauch-Chara K, Hallschmid M, et al. Hypoglycemia unawareness in older compared with middle-aged patient with type 2 diabetes. Diabetes Care. 2009;32:1513-7.

42. Punthakee $Z$, Miller ME, Launer $\sqcup$, et al. Poor cognitive function and risk of severe hypoglycemia in type 2 diabetes: post hoc epidemiologic analysis of the ACCORD trial. Diabetes Care. 2012;35:787-93.

43. Lipska KJ, Warton EM, Huang ES, et al. HbA1C and risk of severe hypoglycemia in type 2 diabetes: the Diabetes and Aging Study. Diabetes Care. 2013:36:3535-42.

44. Danne T, Nimri R, Battelino T, et al. International consensus on use of continuous glucose monitoring. Diabetes Care. 2017:40:1631-40.

45. Kalra S, Mukherjee JJ, Venkataraman S, et al. Hypoglycemia: the neglected complication. Indian J Endocrinol Metab. 2013:17:819-34.

46. 4Noh RM, Graveling AJ, Frier BM. Medically minimising the impact of hypoglycemia in type 2 diabetes: a review. Expert Opin Pharmacother. 2011;12:2161-75.

47. Griesdale DE, de Souza RJ, van Dam RM, et al. Intensive insulin therapy and mortality among critically ill patients: a metaanalysis including NICE-SUGAR study data. Can Med AssoC J. 2009;180:821-7

48. Gill GV, Woodward A, Casson IF, Weston PJ. Cardiac arrhythmia and nocturnal hypoglycaemia in type 1 diabetes - the 'dead in bed' syndrome revisited. Diabetologia. 2009;52:42-5.

49. Allen KV, Frier BM Nocturna hypoglycemia: clinical manifestations and therapeutic strategies toward prevention. Endocr Pract. 2003:9.530-43.

50. Åsvold BO, Sand T, Hestad K, Bjørgaas MR. Cognitive function in type 1 diabetic adults with early exposure to severe hypoglycemia. Diabetes Care. 2010:33:1945-7.

51. Sundberg F Barnard K, Cato A, et al. ISPAD Guidelines. Managing diabetes in preschool children. Pediatr Diabetes. 2017;18:499-517.

52. Anderbro T, Amsberg $\mathrm{S}$, Adamson U, et al. Fear of hypoglycaemia in adults with Type 1 diabetes. Diabet Med. 2010;27:1151-8.

53. Riddle MC. The underuse of insulin therapy in North America. Diabetes Metab Res Rev. 2002;18:S42-S49.

54. Lowes L, Lyne P, Gregory JW. Childhood diabetes: parents' experience of home management and the first year following diagnosis. Diabet Med. 2004;21(6):531-8.

55. Cryer PE. Diverse causes of hypoglycemia-associated autonomic failure in diabetes. N Eng/ J Med. 2004;350: 2272-9.

56. UK Prospective Diabetes Study Group. Intensive bloodglucose control with sulphonylureas or insulin compared with conventional treatment and risk of complications in patients with type 2 diabetes (UKPDS 33). Lancet. 1998;352:837-53.

57. Holman RR, Paul SK, Bethel MA, et al. 10-year follow-up of intensive glucose control in type 2 diabetes. N Engl J Med. 2008;359:1577-89.

58. Gaede P, Lund-Andersen H, Parving HH, Pedersen O. Effect of a multifactorial intervention on mortality in type 2 diabetes. $N$ Engl J Med. 2008;358:580-91.

59. Barnard $\mathrm{K}$, Thomas $\mathrm{S}$, Royle $\mathrm{P}$, et al. Fear of hypoglycaemia in parents of young children with type 1 diabetes: a systematic review. BMC Pediatr. 2010;10:50.

60. Hammer M, Lammert M, Mejías SM, et al. Costs of managing severe hypoglycaemia in three European countries. J Med Econ. 2009:12:281-90.

61. Bronstone A, Graham C. The potential cost implications of averting severe hypoglycemic events requiring hospitalization in high-risk adults with type 1 diabetes using real-time continuous glucose monitoring I Diabetes Sci Technol. 2016;10:905-13.

62. Pawaskar M, Iglay K, Witt EA, et al. Impact of the severity of hypoglycemia on health - related quality of life, productivity resource use, and costs among US patients with type 2 diabetes. J Diabetes Comp. 2018;32(5):451-7.

63. Brod M, Christensen T, Thomsen TL, Bushnell DM. The impact of non-severe hypoglycemic events on work productivity and diabetes management. Value Health. 2011;14:665-71.

64. Brod M, Rana A, Barnett AH. Impact of self-treated hypoglycaemia in type 2 diabetes: a multinational survey in patients and physicians. Curr Med Res Opin. 2012;28:1947-58.

65. Polonsky WH, Fisher L, Hessler D. The impact of non-severe hypoglycemia on quality of life in patients with type 2 diabetes. Jiabetes Comp 2018:32:373-8.

66. Wredling RA, Theorell PG, Roll HM, et al. Psychosocial state of patients with IDDM prone to recurrent episodes of severe hypoglycemia. Diabetes Care. 1992;15:518-2

67. Solli O, Stavem K, Kristiansen IS. Health-related quality of life in diabetes: the associations of complications with EQ-5D scores Health Qual Life Outcomes. 2010;8:18

68. Barnard K, James J, Kerr D, et al. Impact of chronic sleep disturbances for people living with T1 diabetes. I Diabetes SCi Technol. 2016;10:762-7.

69. Evans M, Khunti K, Mamdani M. Health-related quality of life associated with daytime and nocturnal hypoglycaemic events: a time trade-off survey in five countries. Health Qual Life Outcomes. 2013:11:90

70. Geelhoed-Duijvestijn PH, Pedersen-Bjergaard U, Weitgasser $R$, et al. Effects of patient-reported non-severe hypoglycemia on healthcare resource use, worktime loss, and wellbeing in insulin-treated patients with diabetes in seven European countries. J Med Econ. 2013;16:1453-61.

71. Adolfsson P, Parkin CG, Thomas A, Krinelke LG. Selecting the appropriate continuous glucose monitoring system - a practical approach. Euro Endocrinol. 2018;14:24-9.

72. US Food and Drug Administration. Freestyle Libre Flash glucose monitoring system. Summary of Safety and Effectiveness Data (SSED). Available from https://www.accessdata.fda.gov/cdrh docs/pdf16/P160030B.pdf (accessed 21 January 2018).

73. Dexcom G6 continuous glucose monitoring systems user guide. https://s3-us-west-2.amazonaws.com/dexcompdf/G6-CGMUsers-Guide.pdf. Accessed June 2, 2018.

74. Dexcom, Inc. Dexcom G5 Mobile Safety Information. Available at https://www.dexcom.com/safety-information\#dexcom-g5mobile-safety-statement. (accessed 28 January 2018.)

75. US Food and Drug Administration. MiniMed 670G System. Summary of Safety and Effectiveness Data (SSED). Available from https://www.accessdata.fda.gov/cdrh_docs/pdf16/ 160017b.pdf (accessed 21 January 2018).

76. Christiansen MP, Klaff $\amalg$, Brazg RR, et al. A prospective multicenter evaluation of the accuracy of a novel implanted continuous glucose sensor: PRECISE II. Diabetes Technol Ther. 2018;20:197-206

77. Fokkert MJ, van Dijk PR, Edens MA, et al. Performance of the Freestyle Libre Flash glucose monitoring system in patients with type 1 and 2 diabetes mellitus. BMJ Open Diabetes Res Care; $5: \mathrm{e} 000320$

78. US Food and Drug Administration. Summary of Safety and Effectiveness Data (SSED). Dexcom G4 PLATINUM Continuous Effectiveness Data (SSED). Dexcom G4 PLATINUM Continuo us fda.gov/cdrh_docs/pdf12/P120005S018b.pdf (accessed 3 December 2017).

79. Polonsky WH, Hessler D. Perceived accuracy in continuous glucose monitoring: understanding the impact on patients. $J$ Diabetes Sci Technol. 2015;9:330-41

80. Erie C, Van Name MA, Weyman K. Schooling diabetes: use of continuous glucose monitoring and remote monitors in the home and school settings. Pediatr Diabetes. 2018;19: 92-7

81. Lind $\mathrm{M}$, Polonsky W, Hirsch IB, et al. Continuous glucose monitoring vs conventional therapy for glycemic control in adults with type 1 diabetes treated with multiple daily insulin injections: the GOLD randomized clinical trial. JAMA. 2017:317:379-87.

82. Dover AR, Stimson RH, Zammitt NN, Gibb FW. Flash glucose monitoring improves outcomes in a type 1 diabetes clinic Diabetes Sci Technol. 2017:11:442-3.

83. MCKnight JA, Gibb FW. Flash glucose monitoring is associated with improved glycaemic control but use is largely limited to more affluent people in a UK diabetes centre. Diabet Med. 2017;34:732.

84. Ish-Shalom M, Wainstein J, Raz I, Mosenzon O. Improvement in glucose control in difficult-to-control patients with diabetes using a novel flash glucose monitoring device. J Diabetes SC Technol. 2016;10:1412-3.

85. Šoupal J, Petruželková L, Flekac M, et al. Comparison of different treatment modalities for type 1 diabetes, including sensor-augmented insulin regimens, in 52 weeks of follow-up: a COMISAIR study. Diabetes Technol Ther. 2016:18:532-8. 